\title{
The effect of a sitting versus supine posture on normative esophageal pressure topography metrics and Chicago Classification diagnosis of esophageal motility disorders
}

\author{
Yinglian Xiao ${ }^{1,2}$, Andrew Read ${ }^{1}$, Frédéric Nicodème ${ }^{1,3}$, Sabine Roman ${ }^{1,4}$, Peter J. Kahrilas ${ }^{1}$, \\ and John E. Pandolfino ${ }^{1}$ \\ ${ }^{1}$ Department of Medicine, the Feinberg School of Medicine, Northwestern University, Chicago, IL, \\ USA \\ 2Department of Gastroenterology and Hepatology, the First Affiliated Hospital, Sun Yat-sen \\ University, Guangzhou, China \\ ${ }^{3}$ Department of Thoracic Surgery, Université de Montréal, Montréal, QC, Canada \\ ${ }^{4}$ Department of Digestive physiology, Hopisces Civils de Lyon, Lyon I University, Lyon, France
}

\begin{abstract}
Background and aims-Although, the current protocol for high resolution manometry (HRM) using the Chicago Classification is based on the supine posture, some practitioners prefer a sitting posture. Our aims were to establish normative esophageal pressure topography (EPT) data for the sitting position and to determine the effect of applying those norms to Chicago Classification diagnoses.
\end{abstract}

Method-EPT studies including test swallows in both a supine and sitting position of 75 healthy volunteers and 120 patients were reviewed. Integrated relaxation pressure (IRP), distal contractile integral (DCI), contractile front velocity (CFV) and distal latency (DL) were measured and compared between postures. Normative ranges were established from the healthy volunteers and the effect of applying sitting normative values to the patients was analyzed.

Result-Normative values of IRP, DCI and CFV all decreased significantly in the sitting posture. Applying normative sitting metrics to patient studies ( $27 \%$ reduction in IRP (15 to $11 \mathrm{mmHg}$ ), $69 \%$ reduction in DCI (8,000 to $2500 \mathrm{mmHg}-\mathrm{s}-\mathrm{cm})$ ) reclassified 13/120 (11\%) patients as having abnormal EGJ relaxation and 26/120 (22\%) as hypercontractile. Three patients with an abnormal supine IRP normalized when sitting with elimination of a vascular artifact.

Conclusion-Clinical HRM studies should include both a supine and sitting position to minimize misdiagnoses attributable to anatomical factors. However, until outcome studies demonstrating the significance of isolated abnormalities of IRP or DCI in the sitting position are

Correspondence: Yinglian Xiao, MD, Northwestern University, Feinberg School of Medicine, Department of Medicine, 676 St Clair St, Suite 1400, Chicago, Illinois 60611-2951, Tel:312-695-4729, Fax: 312-695-3999, yinglian.xiao@ gmail.com.

Conflict of interest:

John E. Pandolfino [Given imaging and Sandhill Scientific (consulting, educational)]

Sabine Roman [Given imaging (educational)]

No other conflicts for remaining authors (YX, AD, FN, PJK)

Specific author contributions:

Study concept, acquisition of data, analysis, drafting, study supervision : Yinglian Xiao; Acquisition of data and analysis: Andrew Read, Frédéric Nicodèmeand and Sabine Roman; Study concept, acquisition of data, analysis, drafting, study supervision, finalizing the manuscript: Peter J. Kahrilas and John E. Pandolfino 
available, the Chicago Classification of esophageal motility disorders should continue to be based on supine swallows using normative data from the supine posture.

\section{Keywords}

High resolution manometry; position; Chicago classification

\section{Introduction}

The Chicago Classification is the product of a recent global effort to standardize high resolution manometric (HRM) technique and interpretation using esophageal pressure topography (EPT) metrics (1). This scheme was formulated and validated based on a ten 5$\mathrm{ml}$ swallow protocol obtained in a supine position with patients lying flat (0-10 degrees). Normative data for the Chicago Classification were established from two large series of studies done on asymptomatic controls using that protocol $(2,3)$.

Historically, there has been substantial variability among centers in the methodology for clinical esophageal manometry with many centers utilizing a protocol conducted in a sitting position based on the argument that this is more akin to swallowing in real life. That practice has continued with the adoption of high resolution manometry utilizing Chicago Classification criteria for interpretation. However, in so doing, normative data obtained in a supine posture are being utilized to interpret studies conducted in a sitting posture. Previous studies using both conventional and HRM have shown that EGJ morphology and peristaltic variables change with body posture suggesting that cutoffs for abnormality established in the supine position may not be valid in the sitting position (4-9). Specifically, peristaltic amplitude is lower in the sitting position, possibly due to the effect of gravity in promoting esophageal empting $(4,7-9)$. However, to date there has been only one small study aimed at establishing normative values for EPT metrics in the sitting position (8). In that study, Sweis et al reported that the EPT metric for contractile vigor, the distal contractile integral (DCI), was indeed reduced with in the sitting position, consistent with previous conventional manometry data (8). However, Sweis et al also reported that the normal range for the measurement utilized to assess deglutitive esophagogastric junction (EGJ) relaxation, the integrated relaxation pressure (IRP), was greater in the sitting position and this was not consistent with previous data assessing lower esophageal sphincter (LES) relaxation with conventional techniques $(4,9)$. The reason for this conflicting data is unclear.

Thus, the aim of this study was to assess how body position altered the normative ranges and cutoff values of EPT metrics utilized in the Chicago Classification. Furthermore, the effect of hiatal hernia, the most common anatomical abnormality of the EGJ, was assessed to see if this independently altered EPT metrics during positional changes.

\section{Methods}

\section{Study subjects}

Manometric studies were done on 75 healthy volunteers (40 men and 35 women, age: 19-48 yr). Subjects were recruited by advertisement or word of mouth and had been utilized in our previous reports focused on generating normative data ranges in the supine position $(2,3$, 10). Subjects had no history of gastrointestinal symptoms or upper gastrointestinal tract surgery and all were without any significant medical conditions.

To evaluate the effect of changing EPT metrics to those obtained in a sitting position on clinical studies, EPT studies of 120 (41 men and 79 women, age: $16-80$ yr)patients done between October, 2010 and April, 2012 were randomly selected from a consecutive series of 
clinical studies done at the Northwestern Esophageal Center. Among the 120 patients, 40 had hiatal hernia defined by HRM as a separation of at least $2 \mathrm{~cm}$ between the LES and crural contraction and also confirmed on endoscopy reports. HRM metrics were compared in patients with and without hiatus hernia. The study protocol was approved by the Northwestern University Institutional Review Board, and informed consent was obtained from each asymptomatic volunteer while the EPT studies for the 120 patients were part of an IRB approved study to retrospectively analyze all esophageal function studies to study the natural history of esophageal motor disorders.

\section{High Resolution Manometry}

Manometric studies were performed with the patients in the supine position after at least a 6$\mathrm{h}$ fast. The HRM catheters were $4.2 \mathrm{~mm}$ outer diameter solid-state assemblies with 36 circumferential sensors at $1-\mathrm{cm}$ intervals (Given Imaging, Los Angeles, CA). Transducers were calibrated at 0 and $300 \mathrm{mmHg}$ using externally applied pressure. Manometric assemblies were placed transnasally and positioned to record from the hypopharynx to the stomach with at least three intragastric sensors. For the healthy volunteers, the manometric protocol included a 5-min baseline recording and ten 5-ml swallows in the upright position (sitting in a chair at 75-90 degrees). The subjects then assumed a supine position (flat on the back at 0-10 degrees) for an additional ten 5-ml swallows. For the patients, the first ten 5-ml liquid swallows were done supine followed by an additional five 5-ml liquid swallows in a sitting posture.

\section{Data Analysis}

The EPT studies of the volunteers and patients were analyzed with Manoview TM analysis software (Given Imaging, Duluth GA, USA). The EPT metrics analyzed were the IRP, DCI, contractile front velocity (CFV) and distal latency (DL) were measured as previous defined (10).

\section{Statistical analysis}

Data were expressed as median and interquartile range (IQR), and normative data was reported at the 5th and 95th percentile. Metrics were compared between the supine and sitting position using the Wilcoxon Rank Sum test while the Kruskal-Wallis test was used to compare the three groups (asymptomatic volunteers, patients without hernia, and patients with hernia). All P values were two-tailed with the level of significance defined at 0.05 .

\section{Results}

\section{Normative EPT metrics from the $\mathbf{7 5}$ healthy volunteers}

Normative supine and sitting HRM data from the 75 healthy volunteers are shown in Table 1. The IRP, DCI and CFV were all significantly decreased in the sitting position while the DL increased slightly consistent with the decreased CFV. Most notable were that the $95^{\text {th }}$ percentile value of the IRP was reduced by $27 \%$ from $15 \mathrm{mmHg}$ to $11 \mathrm{mmHg}$ and the $95^{\text {th }}$ percentile for the DCI was reduced by $58 \%$ from 4721 to $1991 \mathrm{mmHg}-\mathrm{s}-\mathrm{cm}$ in the sitting position. Overall, few individuals among the 75 exhibited increases in these metrics when sitting: 4 had an increase in IRP and 4 had an increase in DCI.

\section{Changes in EPT metrics among patients}

Tables $2 \mathrm{a}$ and $2 \mathrm{~b}$ summarize the supine and sitting data in the 120 patients without and with hiatus hernia respectively. Consistent with the observation in control subjects, the IRP was significantly reduced in both groups. The DCI was significantly reduced in the non-hernia patients and trended toward a decrease in the patients with hernia, probably not achieving 
significance because of a type II error. When all 120 patients were grouped the DCI was significantly reduced in the sitting posture (1248 vs $937 \mathrm{mmHg}-\mathrm{s}-\mathrm{cm}, \mathrm{p}<0.01)$. In contrast, the CFV did not change significantly with the sitting posture in either patient population and the DL decrease slightly only in the hiatal hernia group.

The magnitude and direction of change in EPT metrics attributed to positional change in both patients and control subjects are presented in Table 3 . There was significantly greater change in each metric comparing the volunteers to the patients but no differences in the magnitude of change between the patients with and without hernia. The effect of positional change was also assessed from the perspective of a differential effect in individuals with normal vs abnormal values for each EPT metric in the supine posture. Both patients with normal IRP $(<15 \mathrm{mmHg})(\mathrm{n}=106)$ and abnormal IRP $(\mathrm{n}=14)$ exhibited the same trend in the EPT metric change as the whole patient group in IRP, CFV, and DCI; the DL decreased slightly only in patients with a normal IRP $(\mathrm{p}<0.01)$. Only three among the 120 patients had a mean DCI greater than 5000 in the supine position and they had the same trend in EPT metric change as the whole patient group with the exception that the CFV increased in one of them in the sitting posture. Five among the 120 patients had a mean CFV greater than 9 $\mathrm{cm} / \mathrm{s}$ in the supine position and the change in EPT metrics in these patients was similar to that observed in those with normal CFV in the supine position.

\section{Effect on diagnosis of using sitting normative cutoff values of EPT metrics}

Applying the $95^{\text {th }}$ percentile of IRP obtained in the sitting position from the 75 healthy volunteers as a cutoff $(<11.0 \mathrm{mmHg}), 24$ patients would be diagnosed as either achalasia or EGJ outflow obstruction (Figure 1). Eleven of these 24 also had an abnormal mean IRP $(>15 \mathrm{mmHg}$ ) in the supine posture so utilizing the sitting posture cutoff did not change their diagnosis. The other 13 patients in this group were discordant with the supine diagnosis (Figure 2); 7 of these had borderline boarderline IRP values in the supine posture (Figure 2, blue) and 6 had a greater IRP value in the upright position attributable to a vascular artifact or hiatus hernia (Figure 2, green). There were an additional 3 patients that were discordant who had abnormal supine IRP values that normalized in the sitting posture also attributable to vascular artifact or hiatus hernia in the supine position (Figure 2, black). Thus, there were 16 discordant cases (13.3\%) between postures and 9 of these were attributable to vascular artifacts or hiatus hernia. In total, $13(10.8 \%)$ patients' diagnoses changed when the new upright IRP cutoff value was applied. Among these, 6 presented with dysphagia, 3 with reflux, 1 with chest pain, 2 with epigastric pain, and one with belching.

Applying the $99^{\text {th }}$ percentile of the DCI obtained in the sitting position from the 75 healthy volunteers as a cutoff (>2500 $\mathrm{mmHg}-\mathrm{s}-\mathrm{cm}$ ) for classifying a jackhammer swallow would substantially increase the number of patients diagnosed with hypercontractile esophagus (Figure 3); 29 of the 120 met that criterion. This is in sharp contrast to the supine position using the $8,000 \mathrm{mmHg}$-s-cm cutoff in which case only 3 patients were diagnosed with hypercontractile esophagus (Figure 4, green). Additionally, there was one subject with a normal supine DCI that became abnormal in the sitting position (Figure 4, blue). In total, 26 (21.7\%) patients' diagnoses changed when the upright cutoff value was applied. Among these, 11 presented with dysphagia, 10 with reflux, 2 with chest pain, 2 with belching, and one with epigastric pain.

In terms of propagation disorders, only 3 of the 120 patients would be diagnosed as rapid contraction with the upright threshold changes including 1 patient with established gastroparesis accompanied by dilated esophagus and another patient with multiple rings in the distal esophagus. The cutoff change for DL did not affect any individual's diagnosis. 


\section{Discussion}

This study assessed the effect of a conducting HRM studies in a sitting as opposed to a supine posture on EPT metrics utilized in the Chicago Classification of esophageal motor disorders (1). The major findings were that, based on an analysis of 75 control subjects, the upper limits of normal for both major metrics were significantly less in the sitting posture; the $95^{\text {th }}$ percentile of normal for the IRP was reduced from 15 to $11 \mathrm{mmHg}$ and the $99^{\text {th }}$ percentile of normal for the DCI was reduced from 8,000 to 2,500 mmHg-s-cm. Based on an analysis of 120 patients, adopting these changes would result in reclassifying normal EGJ relaxation as abnormal in $13(11 \%)$ of cases and normal contractility to hypercontractility in $26(22 \%)$ of cases. Conversely, EGJ relaxation interpreted as abnormal in the supine position was recognized to be normal in the sitting position in 3 cases $(2.5 \%)$ of patients. In contrast to the IRP and the DCI, EPT metrics of propagation were not altered substantially when one considers the effect on diagnosis. In fact, the decreased CFV in the upright position and small increase in DL only changed the diagnosis in 3 patients and this was a change of borderline significance to rapid contraction. These findings demonstrate that cutoff values for abnormal EPT metrics are dependent on the posture in which the HRM study was conducted and suggest that the interpretation of the HRM studies needs to consider these effects.

Our findings are consistent with a previous report on normative ranges in the 'upright' position in terms of the DCI; however, they are conflicting with regard to the IRP and CFV (8). The reason for these differences may relate to differences in the control populations, details of the swallow protocol, or the definition of an 'upright' position. The 'sitting' position utilized in our studies was actually a 70-90 degree angulation. It is possible that patients studied by Sweiss et al were either inclined to less than 70 degrees or were sitting "hunched over" to greater than 90 degrees conceivably increasing intra-abdominal pressure such that it could alter the IRP. Our control subjects underwent 10 swallows in a sitting position followed by 10 supine swallows, whereas the patients performed the supine swallows first. However, the reversed sequence was not associated with any discernible trend of change in EPT metrics making it unlikely that this protocol issue was important. Alternatively, it may just be that our study utilized a greater sample size of control subjects.

Previous analyses of LES relaxation reported reduced values with a more upright position $(11,12)$ and our findings are consistent with that. However, there are no data to support that gravity has a myogenic or neurogenic effect on the sphincter making that an unlikely explanation. More likely, the increased normative range of the IRP in a supine versus a sitting posture is attributable to greater intrabolus pressure in that posture. The IRP is a complex metric, dependent not only on nadir LES relaxation pressure, but also on intrabolus pressure within the hiatal canal during bolus transit and it would be more reasonable to conclude that it is the increased intrabolus pressure rather than the extent of LES relaxation driving the observed systematic increase in IRP. In the upright position, intrabolus pressure may be decreased because gravity assists in esophageal emptying. Additionally, the slower $\mathrm{CFV}$ and longer DL in the upright posture in the healthy volunteers support that the bolus flow rate is likely decreased through the EGJ and this should be associated with a concomitant reduction in the intrabolus pressure based on Newtonian fluid dynamics (13). Thus, using the normative cutoff of $11 \mathrm{mmHg}$ for IRP in the sitting position is justifiable based on its mechanical plausibility.

The reduction in the DCI in the sitting position was substantial, lowering the threshold for defining hypertensive peristalsis ( $95^{\text {th }}$ percentile of normal) to about $2000 \mathrm{mmHg}-\mathrm{s}-\mathrm{cm}$ and for hypercontractile esophagus $\left(99^{\text {th }}\right.$ percentile of normal) to about $2500 \mathrm{mmHg}-\mathrm{s}-\mathrm{cm}$ for. However, the concordance of these diagnoses with those based on supine swallows was poor 
with 29/120 (24\%) of patients meeting the criterion for hypercontractile esophagus in the sitting position compared to only 3 patients meeting the $8,000 \mathrm{mmHg}-\mathrm{s}-\mathrm{cm}$ criteria for the supine position. This may be attributable to the profound effect that gravity has on peristalsis in normal controls and suggests that, unless outcome data demonstrating the significance of isolated abnormalities of DCI in the sitting position become available, the Chicago Classification of hypertensive and hypercontractile esophagus should continue to be based on supine swallows and applying cutoff DCI values obtained from control subjects in a supine posture.

In summary, we observed significant differences in the cutoff values for IRP, DCI and CFV when shifting from the supine to the sitting posture for HRM studies. Among these, changes in IRP had the greatest clinical significance because of potential treatment implications. Applying the cutoffs obtained in the sitting posture caused significant discordance in the diagnoses of achalasia and EGJ outflow obstruction compared to diagnoses made in the supine posture utilizing normative cutoff values obtained in the supine posture. In many instances the discordance in normal/abnormal IRP was related to vascular artifacts or to the effect of a hiatal hernia on intrabolus pressure making it clear that the lower of the supine/ sitting IRP values was the most accurate indicator of EGJ relaxation. However, in other instances borderline normal IRP values in the supine position became borderline abnormal values in the sitting position making the selection of the cutoff value an issue of cardinal importance. Hence, we conclude that the most prudent approach for clinical HRM would include assessment in both a supine and sitting position to minimize misdiagnoses ultimately attributable to anatomical factors. However, until outcome studies demonstrating the significance of isolated abnormalities of IRP in the sitting position become available, the Chicago Classification of EGJ relaxation abnormalities should continue to be based on supine swallows and applying a cutoff IRP value obtained from control subjects in a supine posture.

\section{Acknowledgments}

Funding:

This work was supported by R01 DK079902 (JEP) and R01 DK56033 (PJK) from the Public Health Service.

\section{Reference}

1. AJ Bredenoord MF, Kahrilas PJ, Pandolfino JE, Schwizer W, Smout AJPM. Chicago classification criteria of esophageal motility disorders defined in high resolution esophageal pressure topography. Neurogastroenterol Motil. 2012; 24(S1):57-65. Epub 16 JAN 2012. [PubMed: 22248109]

2. Ghosh SK, Pandolfino JE, Zhang Q, Jarosz A, Shah N, Kahrilas PJ. Quantifying esophageal peristalsis with high-resolution manometry: a study of 75 asymptomatic volunteers. Am J Physiol Gastrointest Liver Physiol. 2006; 290(5):G988-G997. [PubMed: 16410365]

3. Pandolfino JE, Ghosh SK, Zhang Q, Jarosz A, Shah N, Kahrilas PJ. Quantifying EGJ morphology and relaxation with high-resolution manometry: a study of 75 asymptomatic volunteers. Am J Physiol Gastrointest Liver Physiol. 2006; 290(5):G1033-G1040. [PubMed: 16455788]

4. Sears VW Jr, Castell JA, Castell DO. Comparison of effects of upright versus supine body position and liquid versus solid bolus on esophageal pressures in normal humans. Dig Dis Sci. 1990; 35(7): 857-864. [PubMed: 2364840]

5. Tutuian R, Katz PO, Gideon RM, Castell DO. Normal values for esophageal manometry in the sitting position. Am J Gastroenterol. 2001; 96(Suppl):S41.

6. Bernhard A, Pohl D, Fried M, Castell DO, Tutuian R. Influence of bolus consistency and position on esophageal high-resolution manometry findings. Dig Dis Sci. 2008; 53(5):1198-1205. Epub 2007/10/13. [PubMed: 17932767] 
7. Roman S, Damon H, Pellissier P, Mion F. Does body position modify the results of high resolution manometry? Neurogastroenterol Motil. 2010; 22(3):271-275. [PubMed: 19814774]

8. Sweis R, Anggiansah A, Wong T, Kaufman E, Obrecht S, Fox M. Normative values and interobserver agreement for liquid and solid bolus swallows in upright and supine positions as assessed by esophageal high-resolution manometry. Neurogastroenterol Motil. 2011; 23(6):509-e198. Epub 2011/02/24. [PubMed: 21342362]

9. Iwakiri K, Sugiura T, Kotoyori M, Yamada H, Hayashi Y, Nakagawa Y, et al. Effect of body position on lower esophageal sphincter pressure. Journal of gastroenterology. 1999; 34(3):305-309. Epub 1999/08/05. [PubMed: 10433003]

10. Pandolfino JE, Ghosh SK, Rice J, Clarke JO, Kwiatek MA, Kahrilas PJ. Classifying esophageal motility by pressure topography characteristics: a study of 400 patients and 75 controls. Am J Gastroenterol. 2008; 103(1):27-37. [PubMed: 17900331]

11. Bernhard A, Pohl D, Fried M, Castell DO, Tutuian R. Influence of bolus consistency and position on esophageal high-resolution manometry findings. Dig Dis Sci. 2008; 53(5):1198-1205. [PubMed: 17932767]

12. Roman S, Damon H, Pellissier PE, Mion F. Does body position modify the results of oesophageal high resolution manometry? Neurogastroenterol Motil. 2010; 22(3):271-275. Epub 2009/10/10. [PubMed: 19814774]

13. Ghosh SK, Kahrilas PJ, Zaki T, Pandolfino JE, Joehl RJ, Brasseur JG. The mechanical basis of impaired esophageal emptying postfundoplication. Am J Physiol Gastrointest Liver Physiol. 2005; 289(1):G21-G35. [PubMed: 15691873] 

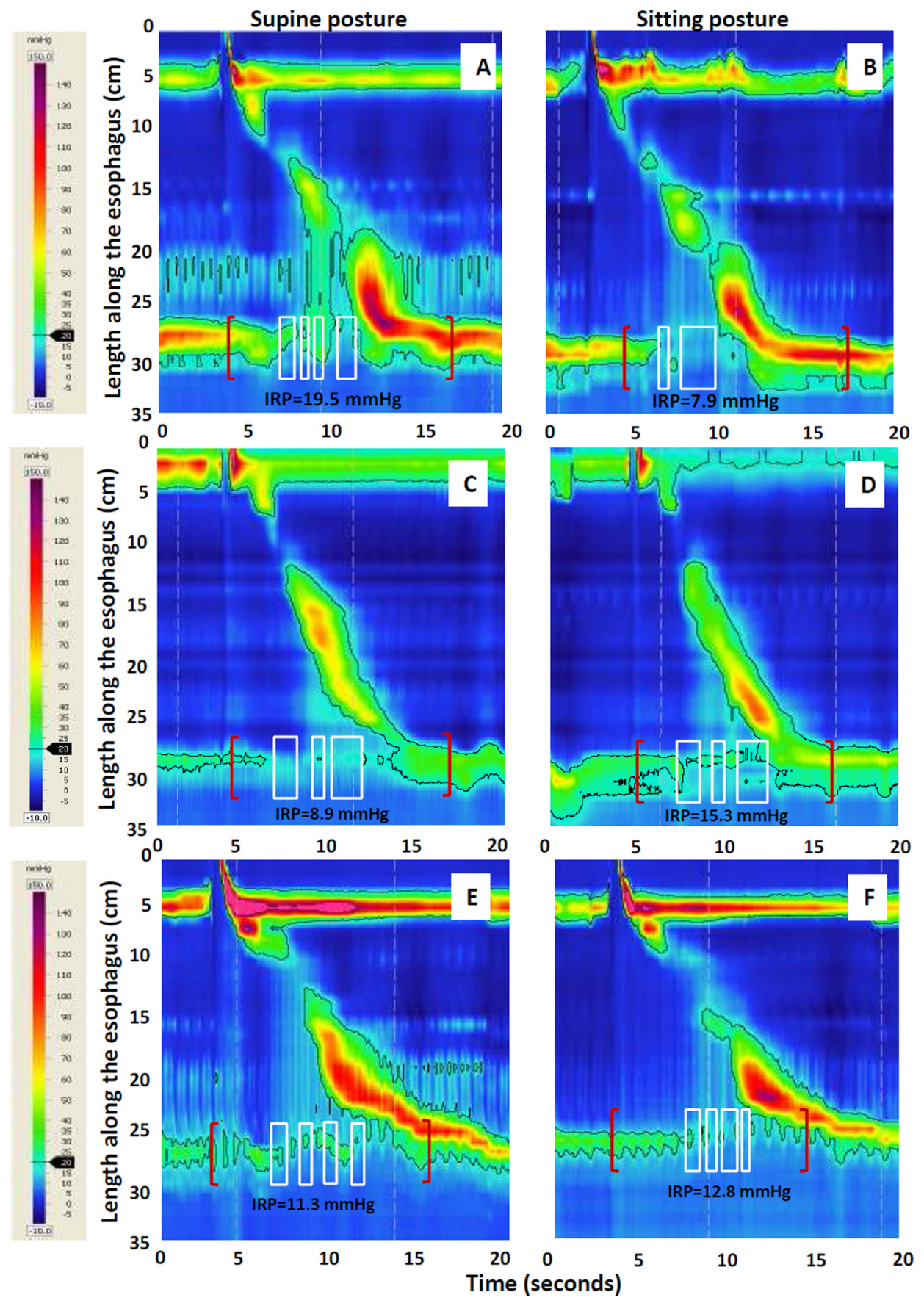

Figure 1.

Examples of the effect of changing the IRP cutoff value on Chicago Classification diagnosis. Panels A and B were swallows from the same patient in supine (left) and sitting (right) position. The mean IRP was $19.5 \mathrm{mmHg}$ in the supine posture but only $7.9 \mathrm{mmHg}$ in the sitting position making the Chicago Classification diagnosis EGJ outflow obstruction when based on the supine position or weak peristalsis when based on the sitting position. Similarly, the patient in Panels C and D was reclassified as EGJ outflow obstruction in the sitting position due to the increased of mean IRP to greater than $11 \mathrm{mmHg}$. Panels $\mathrm{E}$ and $\mathrm{F}$ illustrate a patient in whom EGJ outflow obstruction would be diagnosed in only in the sitting posture due to the lower cutoff value in IRP $(11 \mathrm{mmHg})$. 
$\mathrm{mmHg}$

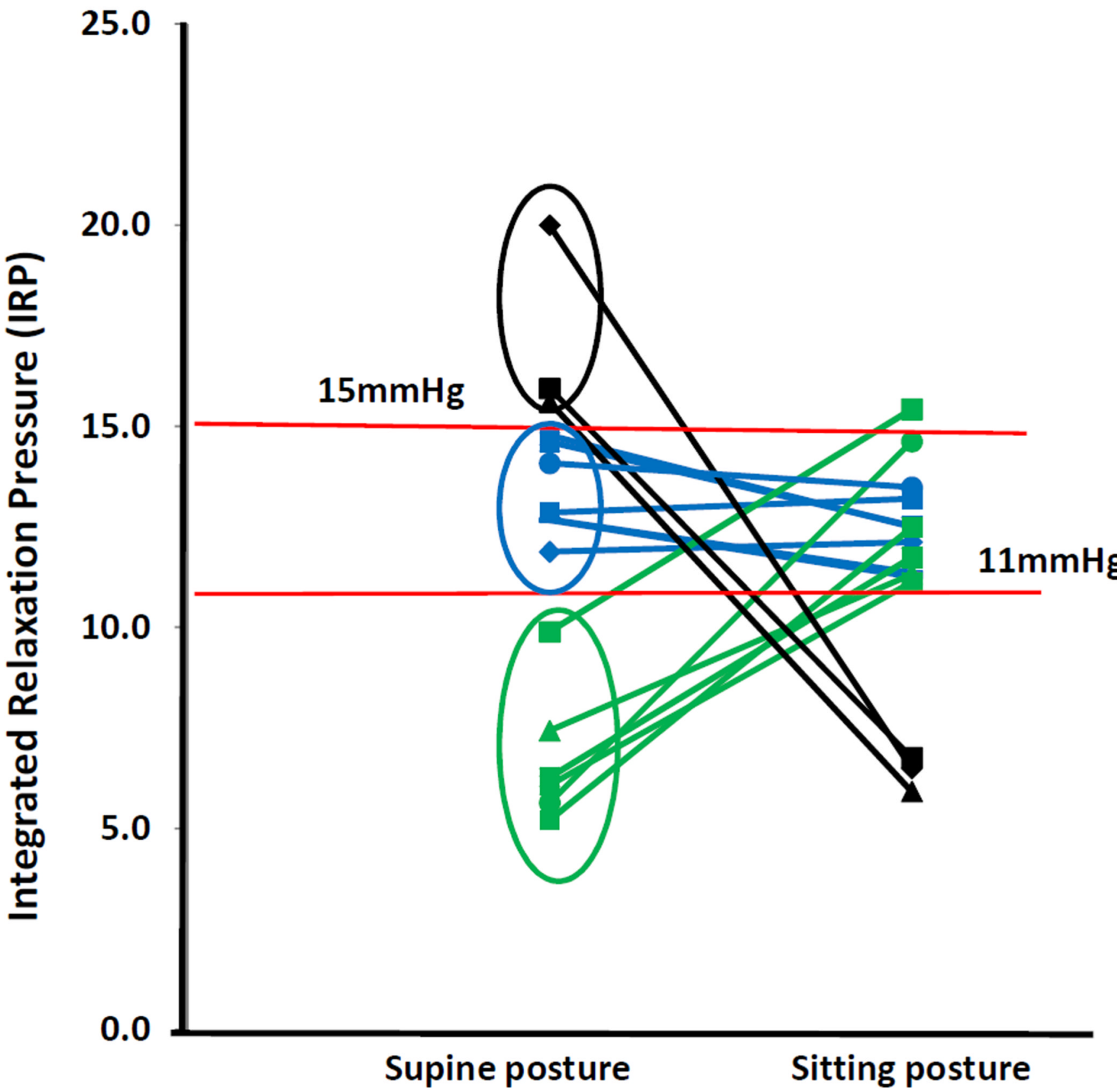

Figure 2.

Individual patients with discordant normal/abnormal mean IRP values depending on whether the supine or sitting cutoff values were applied. Seven patients were borderline abnormal cases when studied supine (blue) that became abnormal in the sitting posture based on the lower cutoff of abnormality $(11 \mathrm{mmHg})$. Six patients had an IRP increase in the sitting position attributable to a vascular artifact or hiatus hernia (green). There were an additional 3 patients with elevated supine values and normal sitting values secondary to vascular artifact or hiatus hernia only in the supine position (black). 

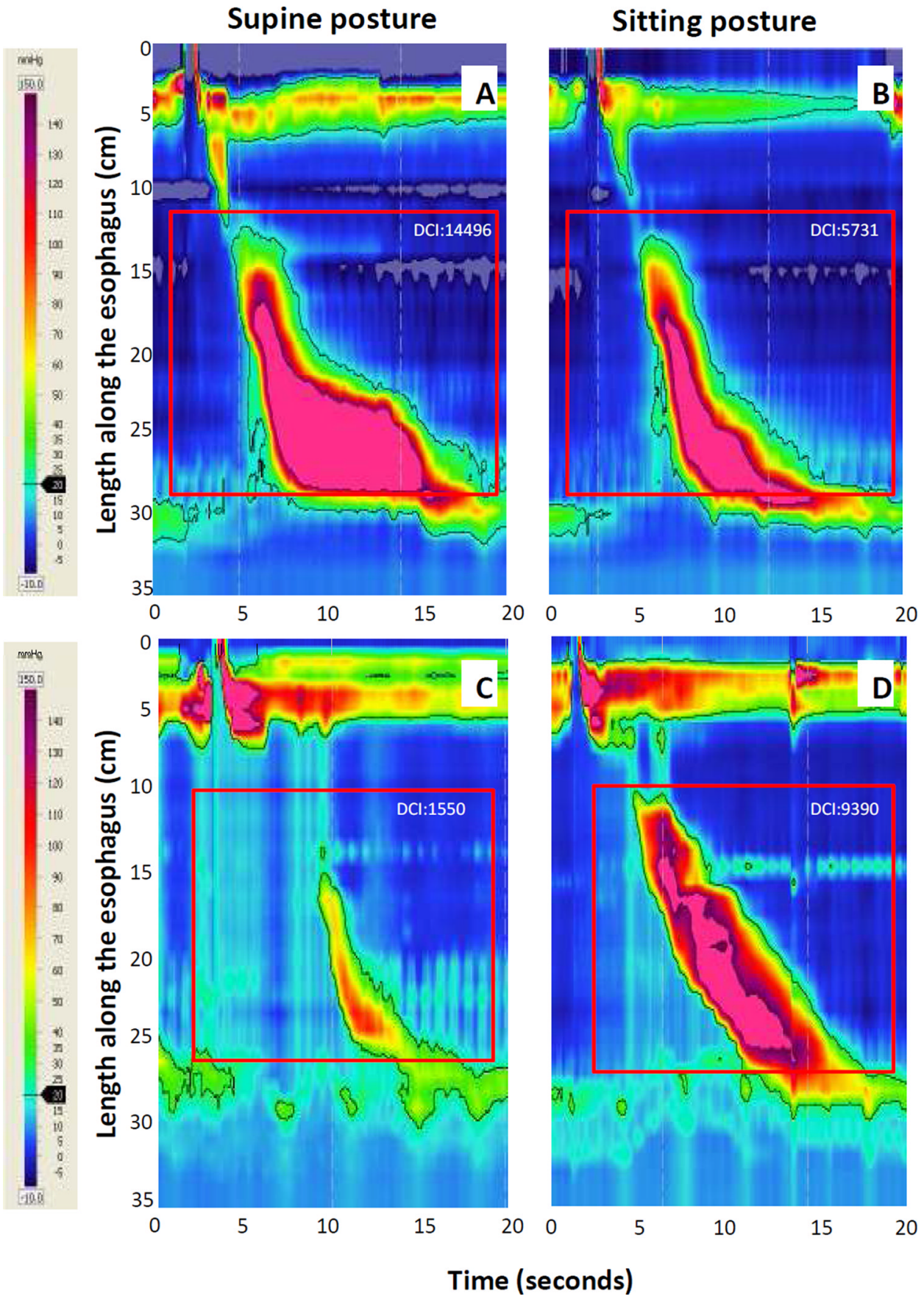

Figure 3.

Effect of the DCI cutoff value on Chicago Classification diagnosis. Panels A and B were swallows obtained from the same patient in supine (left) and sitting (right) position.

Hypercontractility was diagnosed based on the DCI greater than $8000 \mathrm{mmHg}-\mathrm{s}-\mathrm{cm}$ in the supine position; the diagnosis was not changed in the upright position applying the cutoff value of $2500 \mathrm{mmHg}-\mathrm{s}-\mathrm{cm}$, although the maximal DCI changed from 14496 to 5731. Panels $\mathrm{C}$ and D illustrate a patient with normal peristalsis in the supine position with the maximal DCI of less than $5000 \mathrm{mmHg}$-s-cm and hypercontractile esophagus in the sitting position with the maximal DCI of $9390 \mathrm{mmHg}-\mathrm{s}-\mathrm{cm}$. 


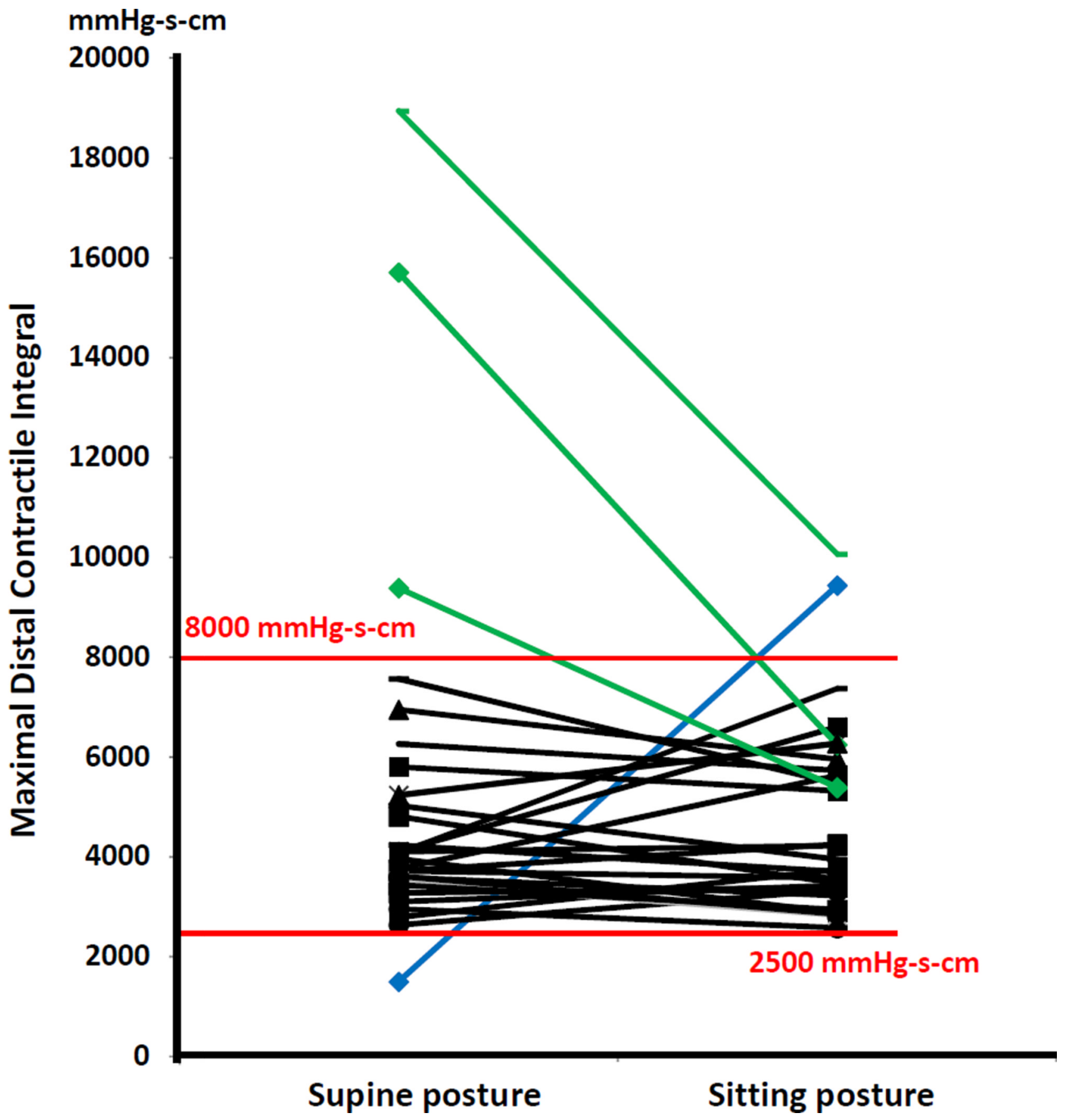

Figure 4.

Individual patients with discordant normal/abnormal maximal DCI values depending on whether the supine or sitting cutoff values were applied. One patient (blue) was reclassified as hypercontractile in the sitting posture due to the increase of maximal DCI to greater than $8000 \mathrm{mmHg}-\mathrm{s}-\mathrm{cm}$. Twenty-five patients were reclassified as "hypercontractile" in the sitting position due to the change of cutoff value (black). Three patients were diagnosed with hypercontractile esophagus in both the supine position and sitting position (green). 
Table 1

Normative HRM data for the 75 patients in supine and sitting position

\begin{tabular}{|c|c|c|c|}
\hline & Supine & Sitting & $\mathbf{p}$ \\
\hline Integrated Relaxation Pressure (mmHg) (Median(IQR)) & $7.9(5.2,9.9)$ & $2.8(1.0,5.3)$ & \multirow[t]{4}{*}{$<0.001$} \\
\hline $5^{\text {th }}$ percentile & 3.1 & 0.2 & \\
\hline $95^{\text {th }}$ percentile & 14.6 & 10.8 & \\
\hline $99^{\text {th }}$ percentile & 18.4 & 13.7 & \\
\hline Distal Contractile Integral (mmHg-s-cm) Median(IQR) & $1612(1066,2128)$ & $698(443,1040)$ & \multirow[t]{4}{*}{$<0.001$} \\
\hline $5^{\text {th }}$ percentile & 448 & 187 & \\
\hline $95^{\text {th }}$ percentile & 4721 & 1991 & \\
\hline $99^{\text {th }}$ percentile & 6478 & 2435 & \\
\hline Contractile Front Velocity (cm/s) Median (IQR) & $4.7(3.7,5.7)$ & $3.9(3.3,4.6)$ & \multirow[t]{4}{*}{$<0.001$} \\
\hline $5^{\text {th }}$ percentile & 2.8 & 2.9 & \\
\hline $95^{\text {th }}$ percentile & 8.9 & 6.1 & \\
\hline $99^{\text {th }}$ percentile & 12.6 & 6.4 & \\
\hline Distal Latency (s) Median (IQR) & $5.8(5.1,6.4)$ & $6.4(5.7,7.2)$ & \multirow[t]{4}{*}{$<0.001$} \\
\hline $5^{\text {th }}$ percentile & 4.3 & 4.9 & \\
\hline $95^{\text {th }}$ percentile & 7.9 & 8.2 & \\
\hline $99^{\text {th }}$ percentile & 9.3 & 9.5 & \\
\hline
\end{tabular}


Table 2

\begin{tabular}{|c|c|c|c|}
\hline \multicolumn{4}{|c|}{ a. EPT metrics of 80 patients without hiatal hernia in the upright and sitting position } \\
\hline & Supine & Sitting & $\mathbf{p}$ \\
\hline Integrated Relaxation Pressure (mmHg) Median (IQR) & $9.2(6.0,13.8)$ & $6.5(3.1,11.3)$ & \multirow[t]{4}{*}{$<0.001$} \\
\hline $5^{\text {th }}$ percentile & 1.1 & 0.2 & \\
\hline $95^{\text {th }}$ percentile & 27.2 & 22.1 & \\
\hline $99^{\text {th }}$ percentile & 35.8 & 26.0 & \\
\hline Distal Contractile Integral (mmHg-s-cm) Median (IQR) & $1331(861,2077)$ & $1062(640,2086)$ & \multirow[t]{4}{*}{$<0.00$} \\
\hline $5^{\text {th }}$ percentile & 220 & 145 & \\
\hline $95^{\text {th }}$ percentile & 4112 & 3379 & \\
\hline $99^{\text {th }}$ percentile & 9111 & 5761 & \\
\hline Contractile Front Velocity $(\mathrm{cm} / \mathrm{s})$ Median (IQR) & $3.9(3.3,4.7)$ & $3.8(3.1,4.8)$ & \multirow[t]{4}{*}{0.331} \\
\hline $5^{\text {th }}$ percentile & 2.1 & 1.8 & \\
\hline $95^{\text {th }}$ percentile & 8.3 & 6.4 & \\
\hline $99^{\text {th }}$ percentile & 15.7 & 29.9 & \\
\hline Distal Latency (s) Median (IQR) & $7.3(6.5,8.0)$ & $7.2(6.7,8.1)$ & \multirow[t]{4}{*}{0.118} \\
\hline $5^{\text {th }}$ percentile & 5.3 & 4.9 & \\
\hline $95^{\text {th }}$ percentile & 10.4 & 8.7 & \\
\hline $99^{\text {th }}$ percentile & 10.6 & 10.5 & \\
\hline
\end{tabular}

\begin{tabular}{|c|c|c|c|}
\hline \multicolumn{4}{|c|}{ b. EPT metrics of 40 patients with hiatal hernia in the upright and sitting position } \\
\hline & Supine & Sitting & $\mathbf{p}$ \\
\hline Integrated Relaxation Pressure (mmHg) Median (IQR) & $5.3(3.6,9.3)$ & $4.6(2.0,7.9)$ & \multirow[t]{4}{*}{0.012} \\
\hline $5^{\text {th }}$ percentile & 0.4 & 0 & \\
\hline $95^{\text {th }}$ percentile & 13.3 & 12.5 & \\
\hline $99^{\text {th }}$ percentile & 14.1 & 14.6 & \\
\hline Distal Contractile Integral (mmHg-s-cm) Median (IQR) & $957(436,1980)$ & $771(366,2216)$ & \multirow[t]{4}{*}{0.059} \\
\hline $5^{\text {th }}$ percentile & 66 & 134 & \\
\hline $95^{\text {th }}$ percentile & 4454 & 5771 & \\
\hline $99^{\text {th }}$ percentile & 15255 & 7122 & \\
\hline Contractile Front Velocity $(\mathrm{cm} / \mathrm{s})$ Median (IQR) & $3.6(2.9,5.4)$ & $3.8(3.0,4.8)$ & \multirow[t]{4}{*}{0.576} \\
\hline $5^{\text {th }}$ percentile & 1.9 & 1.9 & \\
\hline $95^{\text {th }}$ percentile & 13.6 & 12.2 & \\
\hline $99^{\text {th }}$ percentile & 21.6 & 22.5 & \\
\hline Distal Latency (s) Median (IQR) & $7.2(6.5,7.8)$ & $6.7(6.0,7.4)$ & \multirow[t]{4}{*}{0.030} \\
\hline $5^{\text {th }}$ percentile & 5.5 & 5.0 & \\
\hline $95^{\text {th }}$ percentile & 10.1 & 9.0 & \\
\hline $99^{\text {th }}$ percentile & 10.6 & 9.4 & \\
\hline
\end{tabular}


Table 3

Summary of the changes in EPT metrics attributed to positional change in controls, patients without, and patients with hiatal hernia, Median (IQR).

\begin{tabular}{|l|c|c|c|c|}
\hline $\begin{array}{l}\text { HRM metric } \\
\text { difference }\end{array}$ & Controls & $\begin{array}{c}\text { Without hiatal } \\
\text { hernia }^{*}\end{array}$ & $\begin{array}{c}\text { With hiatal } \\
\text { hernia }^{*}\end{array}$ & p \\
\hline Integrated Relaxation Pressure $(\mathrm{mmHg})$ & $-4.3(-6.3,-2.7)$ & $-2.4(-5.1,-0.3)$ & $-1.4(-3.3,0.8)$ & $<0.001$ \\
\hline Distal Contractile Integral (mmHg-s-cm) & $-838(-1264,-480)$ & $-198(-608,11)$ & $-194(-530,19)$ & $<0.001$ \\
\hline Contractile Front Velocity $(\mathrm{cm} / \mathrm{s})$ & $-0.7(-2.5,-0.6)$ & $-0.2(-0.7,0.5)$ & $0(-0.6,0.8)$ & 0.001 \\
\hline Distal Latency (s) & $0.7(0.3,1.1)$ & $-0.1(-1.4,0.3)$ & $-0.5(-1.2,0.3)$ & $<0.001$ \\
\hline
\end{tabular}

$\mathrm{p}<0.05$ versus controls 